\title{
Determination of van der Waals Parameters Using A Double Exponential Potential for Nonbonded Divalent Metal Cations in TIP3P Solvent
}

\section{[Supporting Information]}

Viet Hoang Man, ${ }^{1}$ Xiongwu Wu, ${ }^{*, 2}$ Xibing He, ${ }^{1}$ Xiang-Qun Xie, ${ }^{1}$ Bernard R. Brooks, ${ }^{*, 2}$ and Junmei Wang*,1

${ }^{1}$ Department of Pharmaceutical Sciences and Computational Chemical Genomics Screening Center, School of Pharmacy, University of Pittsburgh, Pittsburgh, PA 15261, USA.

${ }^{2}$ Laboratory of Computational Biology, National Heart, Lung, and Blood Institute, National Institute of Health, Bethesda, Maryland 20892

E-mail:wuxw@nhlbi.nih.gov; brb@mail.nih.gov; junmei.wang@pitt.edu 

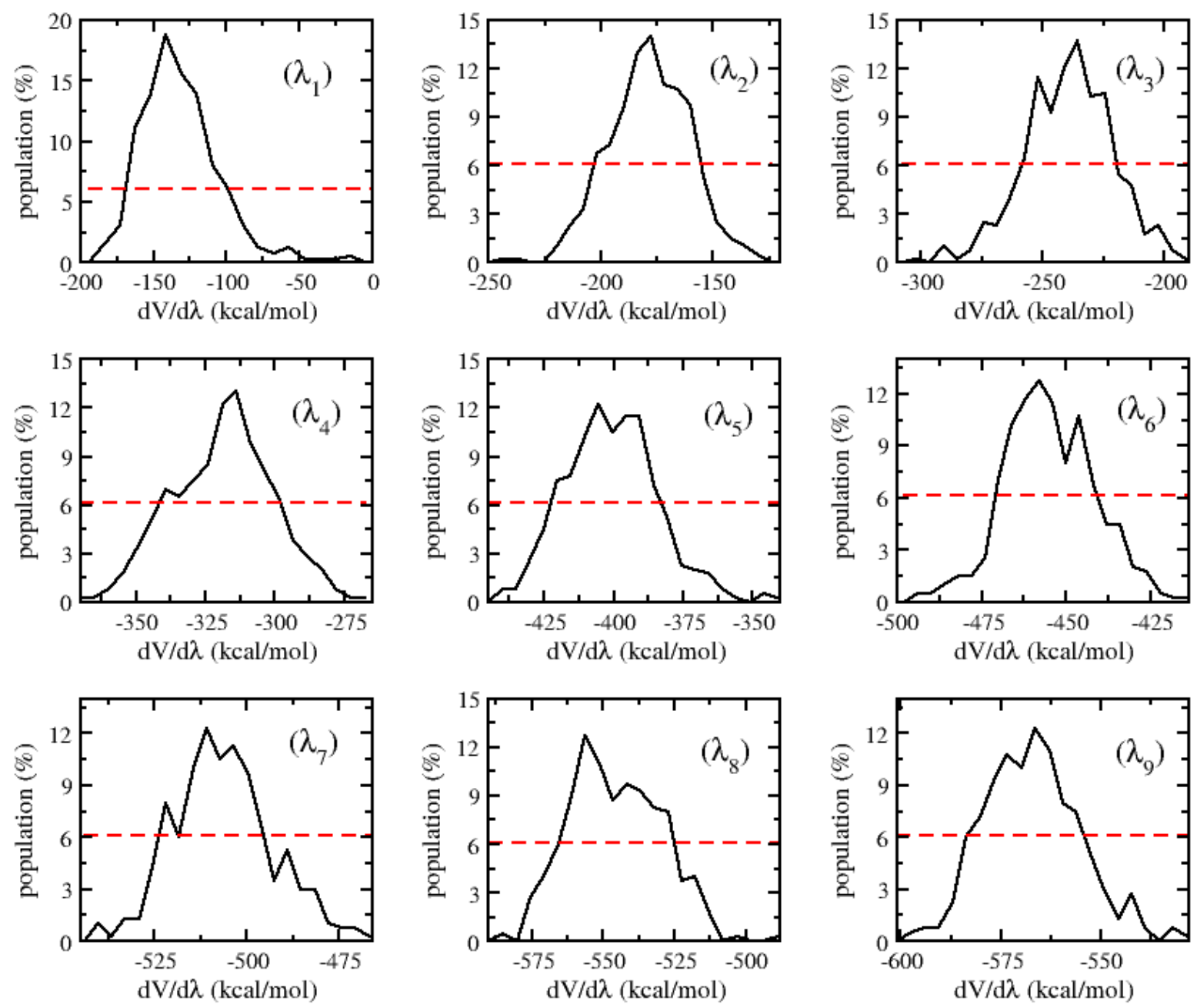

Figure S1: Distribution of $\mathrm{dV} / \mathrm{d} \lambda$ at nine $\lambda_{i}$ windows. The dash red lines indicated the the half of the maximum population value. The data was from the simulations with $(R, \varepsilon)$ pair of $(1 \AA, 10$ $\mathrm{kcal} / \mathrm{mol})$. The similar results were obtained from the simulations with the other $(R, \varepsilon)$ pairs. 

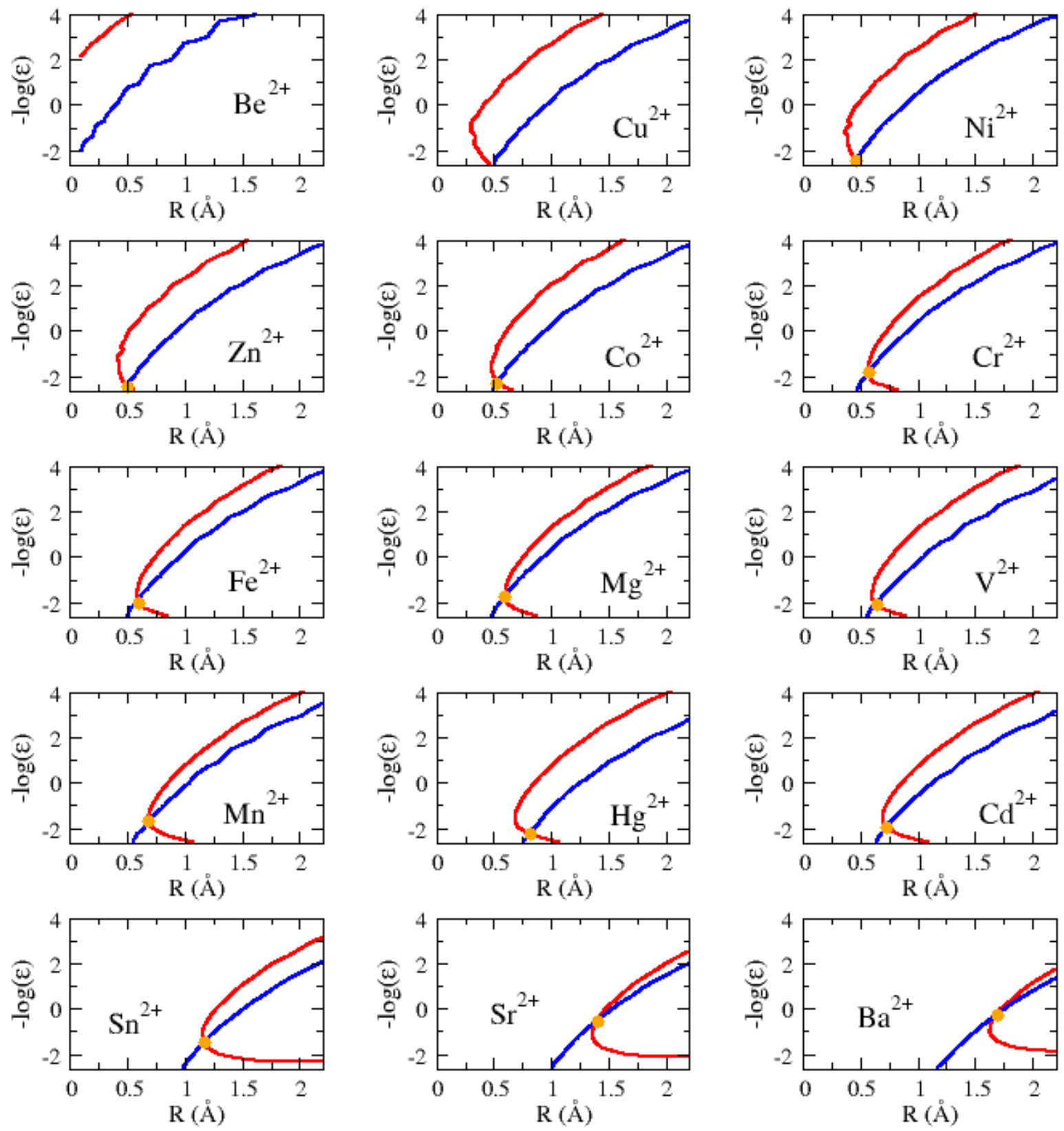

Figure S2: HFE (red line) and IOD (blue line) contour curves of the divalent ions from the VDW parameter coarse-grained for the IPS-DE-TIP3P simulations. The intercross point was shown by orange dot. 

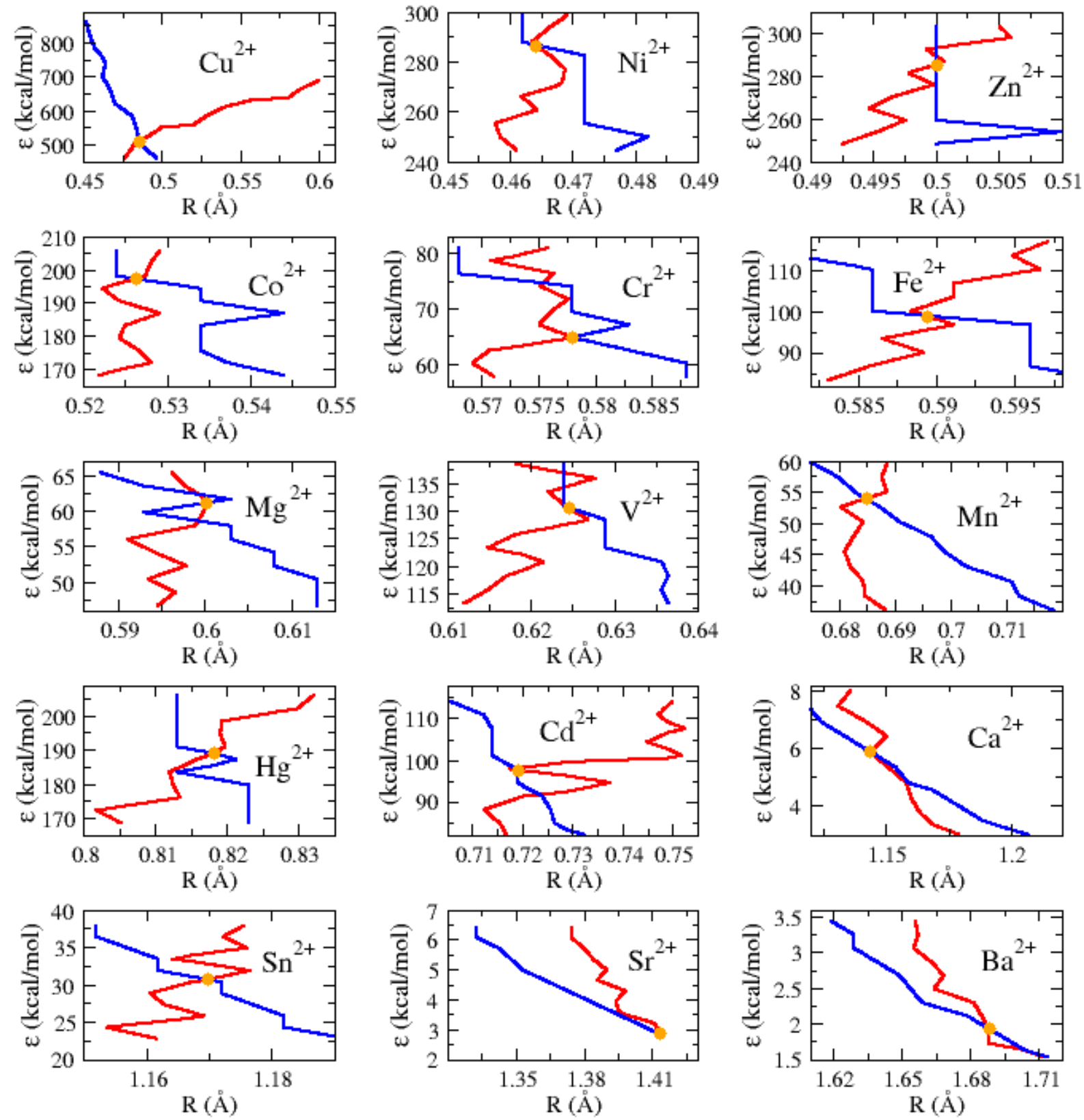

Figure S3: HFE (red line) and IOD (blue line) contour curves of the divalent ions from the VDW parameter fine-grained scan for the IPS-DE-TIP3P simulation. The intercross point was shown by orange dot. 


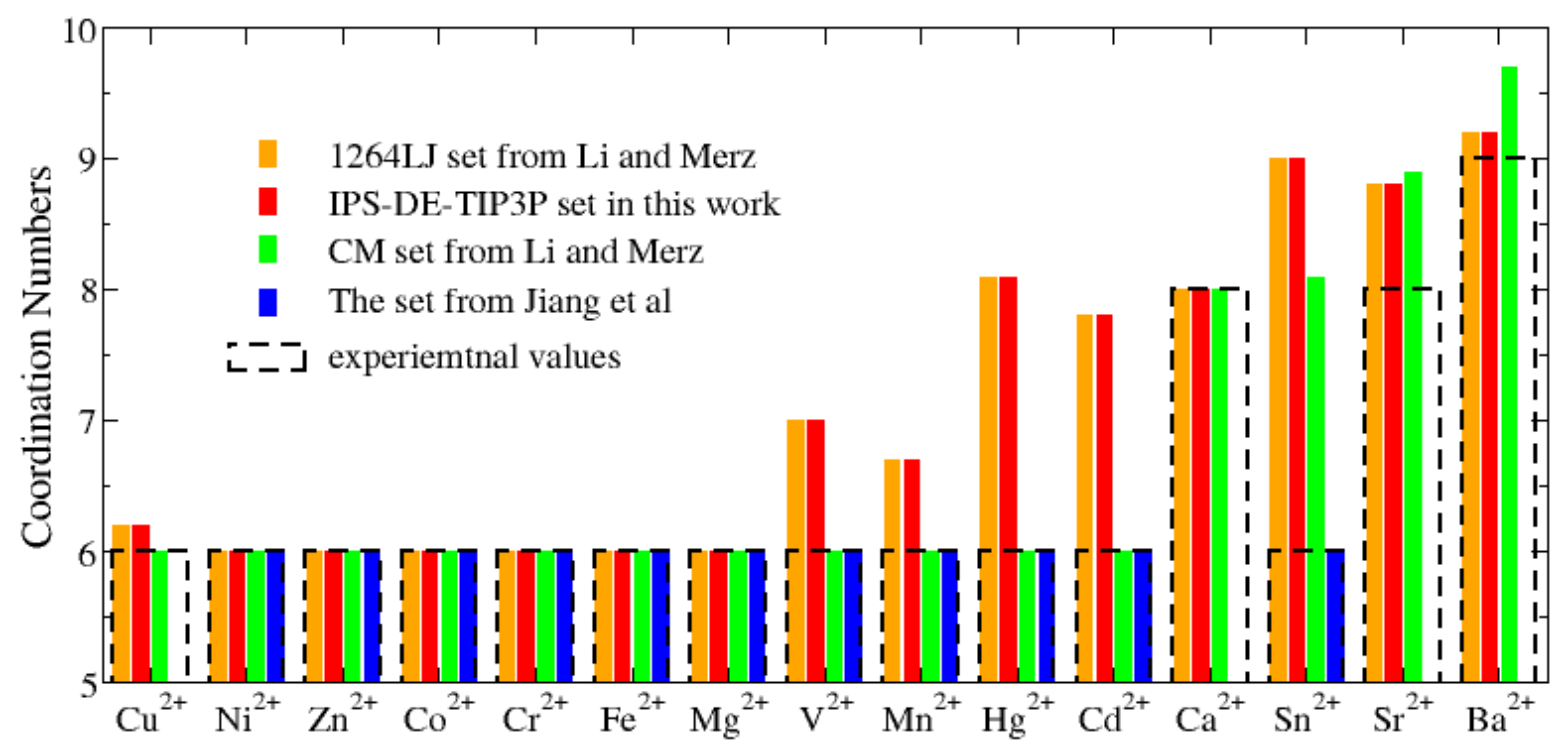

Figure S4: The CN values of the ions from our IPS-DE-TIP3P set, 12-6-4 LJ and CM sets for TIP3P water by Li \& Merz ${ }^{1,2}$, and the set for TIP3P water by Jiang et al. ${ }^{3}$ 


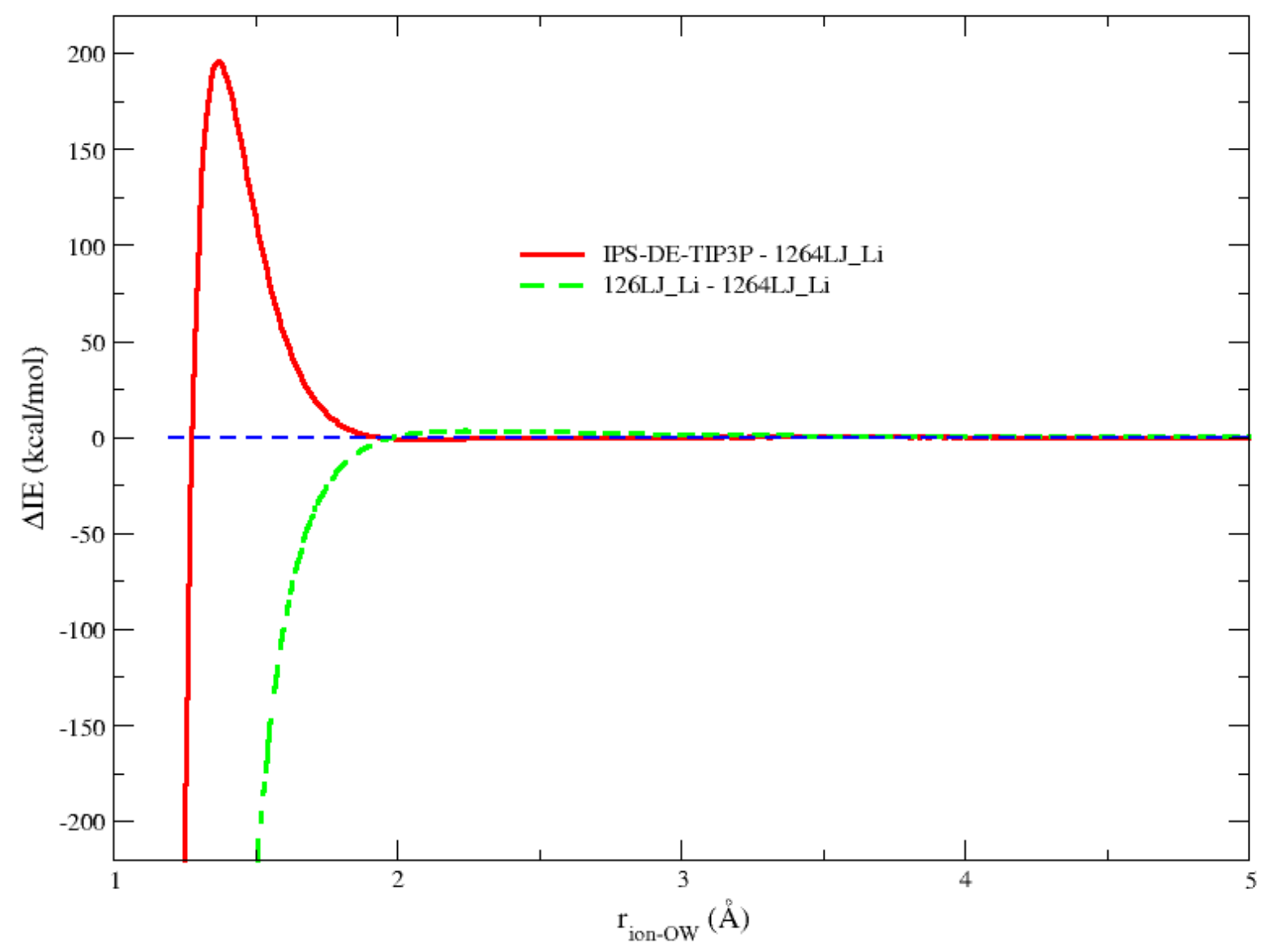

Figure S5: Interaction energy difference using the energies of 12-6-4 LJ model as reference. The data was shown for the interaction between $\mathrm{Mg}^{2+}$ ion and TIP3P water (Figure 7a in main text). 

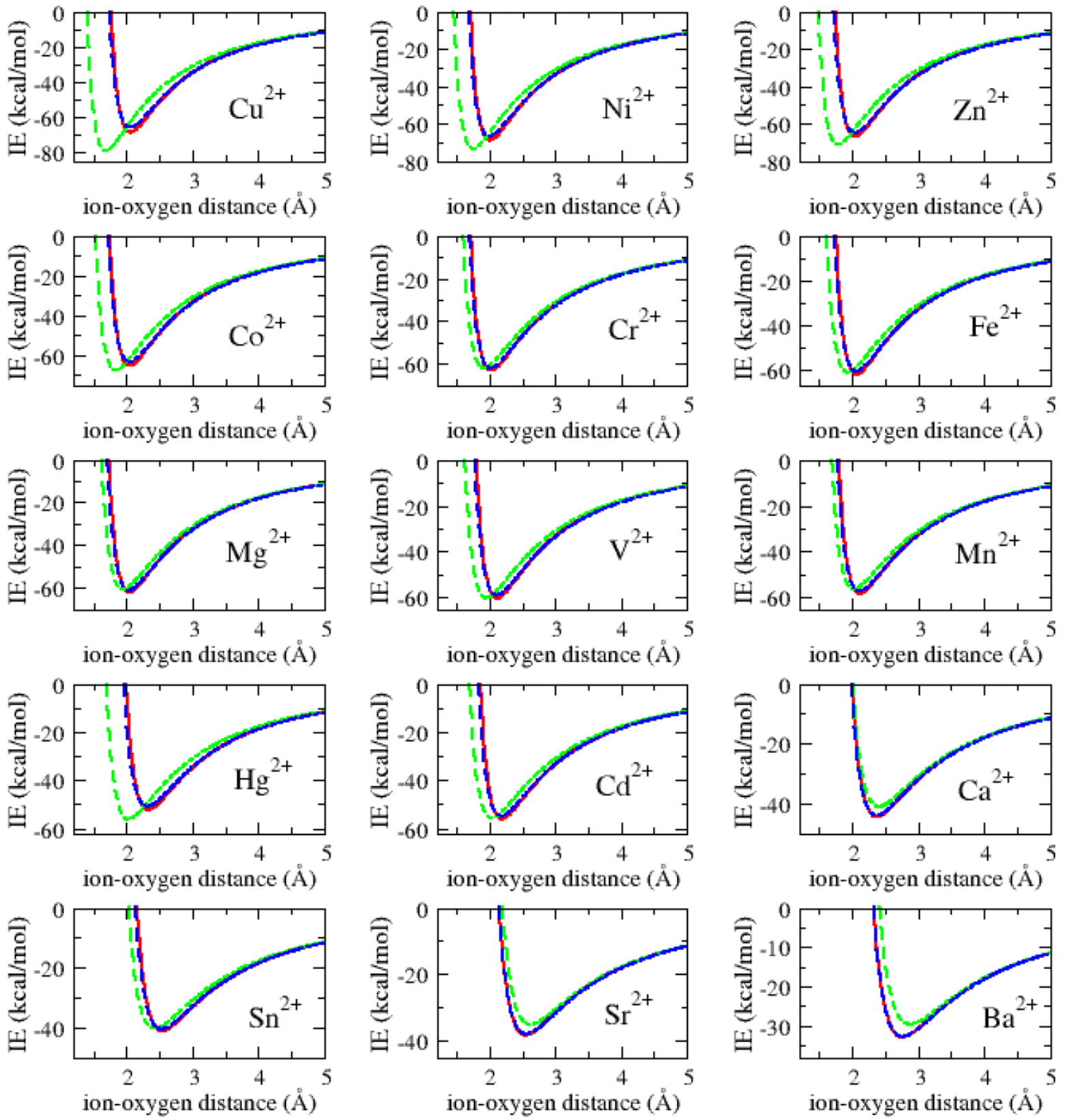

Figure S6: Interaction energy (IE) between TIP3P water and the ion nonbonded models from our IPS-DE-TIP3P set (solid red lines), CM set for TIP3P (dash green lines) and 12-6-4 LJ (dash blue lines) in Li \& Merz works. ${ }^{1,2}$ 


\section{Supporting References}

(1) Li, P.; Kenneth M. Merz, J. Taking into Account the Ion-Induced Dipole Interaction in the Nonbonded Model of Ions. J. Chem. Theory Comput. 2014, 10, 289-297.

(2) Li, P.; Roberts, B. P.; Chakravorty, D. K.; Kenneth M. Merz, J. Rational Design of Particle Mesh Ewald Compatible Lennard-Jones Parameters for +2 Metal Cations in Explicit Solvent. J. Chem. Theory Comput. 2013, 9, 2733-2748.

(3) Jiang, Y.; Zhang, H.; Tan, T. Rational Design of Methodology-Independent Metal Parameters Using a Nonbonded Dummy Model. J. Chem. Theory Comput. 2016, 12, 3250-3260. 